\title{
LA CONSTRUCCIÓN DE LA IDENTIDAD DE LA MUJER CONSUMIDORA DE SUSTANCIAS CONSIDERADAS ILEGALES Y EL ROL DE MADRE
}

Gian Piero Turchi

Martina Sarasin

Simona Fanelli

\section{Introducción}

El presente artículo es una aportación al estudio de la construcción de la identidad de la mujer, desde la perspectiva del enfoque cognoscitivo del Paradigma Narrativista. Tal paradigma se basa en un nivel de realismo conceptual según el cual el conocimiento no se definiría como una plasmación de la realidad (como se entiende desde una perspectiva del realismo monista en la que el dato o evidencia es inobservable, pero potencialmente conocible), y tampoco desde una perspectiva en la que el investigador puede aproximarse al dato únicamente a través del "filtro" de la Teoría (realismo hipotético). Sin embargo, a un nivel de realismo conceptual, nivel en el cual se inscribe el objeto de esta investigación psicológica, el conocimiento genera la realidad, es decir el dato puro no existe, independientemente del sistema de categorización y de conocimiento utilizado para conocerlo como tal. Por otra parte, lo que se describe se hace real en sus efectos pragmáticos, en virtud del acto cognoscitivo que viene puesto en juego. En ese sentido, de acuerdo con el nivel de realismo conceptual, el objeto de estudio se constituye mediante un modelo cognoscitivo que ponen en marcha las personas investigadas, mediante la construcción de una realidad específica y no por los contenidos en sí. Un tema fundamental en el Paradigma Narrativista son las prácticas discursivas, definibles como el conjunto de modalidades cognoscitivas que contribuyen a generar, construir y mantener una configuración de la 
realidad (la "conocida"). Por tanto, desde una perspectiva narrativista, se describirán los procesos cognoscitivos (que son la base de la realidad, objeto de la investigación). Concretamente, la presente investigación plantea como objetivo describir las modalidades cognoscitivas que contribuyen a la construcción de la identidad en la mujer consumidora de sustancias ilegales; en particular intenta describir si los modelos cognoscitivos cambian en el momento en que una mujer decide ser madre y, en caso afirmativo, cómo cambian.

\section{Grupo objeto de investigación}

El grupo objeto de investigación esta constituido por 80 personas $^{1}$, todas mujeres de edades comprendidas entre 18 y 44 años y distinguidas de acuerdo a dos variables: el consumo de sustancias consideradas ilegales ${ }^{2}$ y el estado de embarazo.

En lo específico, el grupo de investigación esta subdivido en: 25 mujeres consumidoras no embarazadas, 6 consumidoras en estado de embarazo, 24 mujeres no consumidoras no embarazadas y 25 no consumidoras de sustancias ilegales en estado de embarazo.

El grupo objeto de investigación es heterogéneo en relación a la profesión y al contexto socio-económico.

Todas las mujeres embarazadas que han sido entrevistadas están en su primer embarazo, mientras las mujeres del grupo "no embarazadas" no tienen hijos.

En lo específico, el grupo de mujeres consumidoras de sustancias ilegales está compuesto de mujeres en tratamiento con metadona en los Servicios para las Drogodependencias, que han hecho uso en preponderancia de la heroína, en algunos

\footnotetext{
${ }^{1}$ La aplicación de los protocolos de investigación se ha realizado durante el segundo semestre del año 2004.

${ }^{2}$ Las mujeres consumidoras entrevistadas en el momento de la aplicación del protocolo de investigación se beneficiaban del tratamiento de metadona en los servicios para drogodependientes que han hecho uso de heroína y en algunos casos también de otras sustancias en asociación (como, por ejemplo, cocaína).
} 
La construcción de la identidad de la mujer consumidora de sustancias ilegales...

casos de manera exclusiva, en otros en asociación con otras sustancias consideradas ilegales (cocaína, cannabis, etc.).

Las mujeres consumidoras entrevistadas toman contacto con los Servicios para las Drogodependencias exclusivamente por motivos relacionados con la suministración de metadona y a los controles médicos relacionados; sólo esporádicamente, y no de forma continuada, utilizan servicios de sostén psicológico o coloquios con el personal del servicio.

Ese modo de contacto entre mujer consumidora y servicio permite decir que puesto que las mujeres no están insertas en un contexto institucional, las voces narradoras presentes en el servicio no contribuyen de forma predominante a la definición de la identidad de las mujeres mismas, diferentemente de cómo podría ser en el caso de la comunidad terapéutica donde los contactos con el personal es cotidiano.

Se pueden realizar hipótesis además sobre que sean el contexto familiar y social los que contribuyen mayormente a la construcción y mantenimiento de la realidad que se quiere investigar, o sea, la realidad de la mujer embarazada consumidora de sustancias ilegales.

\section{Metodología}

De acuerdo con los temas teórico-epistemológicos expuestos, la metodología de investigación adoptada prevé el análisis estadístico del texto producido por las mujeres entrevistadas.

Por ello, desde un punto de vista metodológico se procedió a la construcción de un protocolo de investigación de preguntas abiertas. La elección de la pregunta abierta se establece, a su vez, en términos de coherencia con los temas teóricos adoptados, ya que establece en el sujeto que responde la condición de explicitar las propias teorías sobre la realidad producida por la pregunta sin tener que elegir entre un elenco de opciones restringido y preestablecido; y, por otra parte, la formulación del texto ofrecido como respuesta, permite al investigador reemplazar las modalidades 
cognoscitivas que el sujeto que habla utiliza en su aproximación a la realidad especifica indagada.

El cuestionario de investigación suministrado está compuesto de 7 preguntas abiertas comunes a los 4 grupos de investigación; además hay dos preguntas específicas respeto a la condición de embarazo y al consumo de sustancias consideradas ilegales, respectivamente al grupo de las mujeres embarazadas y al grupo de mujeres consumidoras.

Las preguntas hechas quieren recoger, por un lado, las descripciones que las mujeres hacen de ellas mismas (en calidad de persona, en calidad de madre y en calidad de consumidora de sustancias ilegales) y de los demás (mujeres consumidoras y mujeres consumidoras embazadas); por otro lado, las narraciones (o sea, las teorías que las entrevistadas atribuyen a la gente común y a los propios familiares).

De acuerdo con los temas teóricos adoptados, la presente investigación refleja en el texto producido por las personas entrevistadas el propio objeto de investigación. Por tanto, desde esta óptica, el análisis del texto es la estrategia de elección en cuanto permite notar las teorías frente a las cuales las entrevistadas producen las respuestas, o sea, construyen y mantienen la propia realidad, y de esta forma se utiliza un método coherente y adecuado a los temas teóricos declarados.

A partir de las respuestas aportadas por el grupo objeto de investigación, se efectuó el análisis del texto con el auxilio del programa estadístico Spadt-t que permite de asociar a cada entrevistado datos externos que pueden ser utilizados come variables nominales. A través del procedimiento ASPARM, a cada palabra se le asigna una posición sobre los ejes cartesianos en relación a su posición respecto a todas las demás palabras (o sea, a la frecuencia con la cual las palabras aparecen relacionadas en la producción del texto).

A partir de los datos textuales obtenidos de esta manera, fue posible individuar los repertorios discursivos utilizados por el grupo objeto de indagación, y por consiguiente describir la forma de conocer la realidad, discursivamente entendida y con 
La construcción de la identidad de la mujer consumidora de sustancias ilegales...

valor de verdadero, que genera, construye y mantiene la realidad de "mujer consumidora de sustancias ilegales" y de "madre".

\section{Exposición y discusión de los resultados}

\subsection{Criterios para la descripción y la discusión de los datos}

Se expondrán a continuación los resultados de la investigación realizada. Por cada pregunta serán descritos los resultados con la ayuda de una tabla en la cual se sintetizarán los repertorios que se han generado. Para cada repertorio serán indicadas también las áreas semánticas que las caracterizan y que en este estudio llamaremos archipiélagos de significado, junto con las formas gráficas más representativas.

Para facilitar la lectura de los resultados, recogemos a continuación un glosario de repertorios que surgen en el proceso de investigación.

\begin{tabular}{|l|l|}
\hline Repertorio Discursivo & Descripción \\
\hline Repertorio del Juicio & $\begin{array}{l}\text { Modalidad discursiva que utiliza una persona, } \\
\text { cuando connota, valorando respecto a algo, } \\
\text { empleando teorías personales; un nivel no } \\
\text { descriptivo pero de connotación, un punto de } \\
\text { vista, o una valoración personal. }\end{array}$ \\
\hline Repertorio de la Proyección al Futuro & $\begin{array}{l}\text { Modalidad discursiva que describe un escenario } \\
\text { futuro, anticipable a partir del conocimiento de } \\
\text { la situación actual. }\end{array}$ \\
\hline Repertorio de las Comparaciones & $\begin{array}{l}\text { Modalidad discursiva que yuxtapone dos } \\
\text { realidades, con el objeto de realizar una } \\
\text { evaluación de las semejanzas, de la afinidad y } \\
\text { las diferencias, manteniéndolas de este modo } \\
\text { distintas. La realidad viene construida y } \\
\text { mantenida a través de una evaluación que } \\
\text { implica la comparación/parangón (comparación } \\
\text { /confrontación) }\end{array}$ \\
\hline
\end{tabular}


Repertorio de la Justificación

Modalidad discursiva que se refiere a las razones de base, que se encuentran en una situación o comportamiento, y que permiten justificar el argumento del discurso. La práctica de esa modalidad discursiva se basa en el mantenimiento del estado actual de las cosas, en cuanto que el empleo de justificaciones, legitima el estado de la situación y no permite poner en juego, otras modalidades de llevar o modificar lo que está ocurriendo.

Tab. 1. Glosario de repertorios

\subsection{Descripción de las respuestas aportadas por la pregunta “¿Cómo te describirías en general como persona?”}

La pregunta propuesta intenta describir las modalidades cognoscitivas utilizadas por las mujeres en la descripción de sí mismas como personas.

A partir del análisis del texto de las respuestas aportadas por las preguntas presentadas, se plantea cómo las entrevistadas utilizan un único repertorio discursivo, el repertorio de juicio y 4 archipiélagos de significado, presentado como resumen en la tabla siguiente.

\begin{tabular}{|l|l|l|}
\hline \multirow{2}{*}{ Repertorio Discursivo } & Archipiélago de significado & Formas gráficas \\
\hline \multirow{3}{*}{ Juicio } & Determinación & Determinada, testaruda \\
\cline { 2 - 3 } & Satisfacción personal & Satisfecha, con éxito \\
\cline { 2 - 3 } & Sensibilidad & Paciente, tímida, sensible \\
\cline { 2 - 3 } & Apertura a los demás & Altruista, sociable, abierta \\
\hline
\end{tabular}

Tab. 2. Repertorios discursivos

A través de la utilización del "Repertorio del Juicio", las entrevistadas se describen mediante teorías personales, es decir, un testimonio a un nivel no descriptivo 
La construcción de la identidad de la mujer consumidora de sustancias ilegales...

pero connotante, un punto de vista o una evaluación personal y por ello una realidad inmutable y no sujeta a cambios. En particular, considerando los contenidos ya expresados, se muestra claramente el empleo de archipiélagos de significado que conducen, por una parte, a características que podríamos definir distintivas y, por otra, a alcanzar los propios objetivos. Las entrevistadas usan, de hecho, los archipiélagos de la determinación, archipiélago caracterizado por la forma gráfica "determinada" y "testaruda"; de la satisfacción personal caracterizado por las formas gráficas "satisfecha" y "éxito"; de la sensibilidad, representado por las formas gráficas "paciente", "tímida" y "sensible" y finalmente, del archipiélago de la apertura hacia los demás, localizable en las formas gráficas "altruista", "sociable”, "abierta".

En referencia a las variables consideradas, se observa cómo el grupo de mujeres embarazadas y el de las mujeres no tóxico-dependientes, se asocian a repertorios de la sensibilidad, de la determinación y de la apertura hacia los demás, y en contra, no se asocian directamente entre las entrevistadas al discurso de los grupos de mujeres embarazadas y no embarazadas.

\subsection{Descripción de las respuestas aportadas por la pregunta “¿Cómo te describirías como madre en el futuro?”}

La pregunta planteada intenta describir las modalidades cognoscitivas utilizadas por las mujeres investigadas en la descripción de proyecciones futuras de sí mismas en el rol de madre.

A partir del análisis del texto de las respuestas aportadas por las preguntas realizadas, se muestra cómo las entrevistadas utilizan dos repertorios discursivos: el repertorio del juicio y el repertorio de la proyección en el futuro y 4 archipiélagos de significado, sintetizados en la siguiente tabla. 


\begin{tabular}{|l|l|l|}
\hline \multirow{2}{*}{ Repertorio Discursivo } & Archipiélago de significado & Formas gráficas \\
\hline \multirow{2}{*}{ Juicio } & Comportamiento protector & Muy protectora, celosa \\
\cline { 2 - 3 } & Comportamiento permisivo & Disponible, permisiva \\
\cline { 2 - 3 } & Estado de ánimo & Feliz, temerosa, serena \\
\hline Proyección en el futuro & Intento/propósito & $\begin{array}{l}\text { Intentaré hacer lo mejor que } \\
\text { puedo }\end{array}$ \\
& & \\
\hline
\end{tabular}

Tab. 3. Repertorios discursivos

Al igual que en la pregunta precedente que solicitaba aportar una descripción de sí misma como persona, también en las respuestas a esas preguntas (que a su vez pedían una descripción de sí en el rol de madre), las entrevistadas utilizan la modalidad cognitiva del juicio, aportando entonces una evaluación a partir de teorías personales. Sin embargo, en términos de contenido, las entrevistadas hacen referencia en perspectiva futura al comportamiento que adoptarán con referencia a su propio hijo. Del análisis del texto se revelan, de hecho, dos archipiélagos de significado: el archipiélago del comportamiento protector, caracterizado de las formas gráficas "muy protectora" y "celosa" y el archipiélago del comportamiento permisivo, caracterizado de las formas gráficas "disponible" y "permisiva".

Junto al repertorio del juicio las entrevistadas utilizaron el repertorio de la proyección en el futuro, a través del cual se describen en un escenario futuro, con contenidos que recuerdan a una declaración de intenciones mediante el archipiélago del propósito, caracterizado por formas gráficas como las de "buscaré”,o "haré lo mejor que puedo".

En cuanto a las variables consideradas, es posible evidenciar cómo todas las modalidades de las variables consideradas resultan estar asociadas con el repertorio del juicio. El repertorio de la proyección futura viene utilizado en modo característico por el grupo de las mujeres embarazadas, sean o no consumidoras de sustancias. 
La construcción de la identidad de la mujer consumidora de sustancias ilegales...

\subsection{Descripción de las respuestas aportadas a la pregunta “¿Cómo te describirías embarazada?”}

La pregunta propuesta intenta describir las modalidades cognoscitivas utilizadas por las mujeres en las descripciones de sí mismas, en calidad de mujeres embarazadas.

En el análisis del texto de las respuestas entregadas a dicha pregunta, se observa cómo las entrevistadas utilizan dos repertorios discursivos: el repertorio del juicio y el repertorio de la proyección futura y 4 archipiélagos de significado, como se resumen en la tabla siguiente.

\begin{tabular}{|l|l|l|}
\hline \multirow{2}{*}{ Repertorio Discursivo } & Archipiélago de significado & Formas gráficas \\
\hline \multirow{2}{*}{ Juicio } & Estados de ánimo & Relajada, conciente \\
\cline { 2 - 3 } & Experiencia mística & $\begin{array}{l}\text { Emocionada, experiencia } \\
\text { milagrosa }\end{array}$ \\
\cline { 2 - 3 } & Vivencia física & Temerosa, ansiosa \\
\hline Proyección futura & Cambio & Futuro, la vida cambia \\
\hline
\end{tabular}

Tab. 4. Repertorios discursivos

Las mujeres entrevistadas dan una descripción de sí, en cuanto mujeres embarazadas, a través de las modalidades cognoscitivas del juicio y de la proyección en el futuro. Los mismos repertorios son utilizados en la descripción de sí mismas como madres (y por tanto con una proyección de futuro). La diferencia entre estas dos respuestas está nuevamente en el nivel de los contenidos. Las mujeres que se describen en virtud del actual estado de embarazo, utilizan un juicio de sí mismas en relación al propio estado de ánimo y la propia experiencia física (su vivencia), mientras que su proyección futura asume un matiz de significado en relación al cambio de la propia vida. De hecho, desde el punto de vista semántico, el repertorio del juicio está caracterizado por el archipiélago de los estados de ánimo y por las formas gráficas "relajada" y "experiencia milagrosa", de la experiencia mística, como de las formas 
gráficas "emocionada" y "permisiva" y del archipiélago de la vivencia física, caracterizado de las formas gráficas "temerosa" y "ansiosa". Por otra parte, el repertorio de la proyección en el futuro está caracterizado tanto por el archipiélago del cambio, como por las formas gráficas de "futuro" y "cambio de vida".

En relación a las variables consideradas, se muestra cómo el grupo de las personas tóxicodependientes utilizan de modo característico el repertorio de la proyección en el futuro y el archipiélago de los estados de ánimo, propio del repertorio del juicio. Característicos del grupo de las personas no consumidoras son los archipiélagos de la experiencia mística y de la vivencia física.

\subsection{Descripción de las respuestas entregadas frente a la pregunta “¿Cómo describirías una mujer tóxico-dependiente?”}

La pregunta intentaba describir las modalidades cognoscitivas usadas por las mujeres entrevistadas durante la descripción de una mujer tóxico-dependiente.

El análisis del texto de las respuestas a esta pregunta muestra cómo las entrevistadas utilizan tres repertorios discursivos, el repertorio de la justificación, el del juicio y el repertorio de la comparación y 6 archipiélagos de significado, que se muestran de forma resumida, en la siguiente tabla.

\begin{tabular}{|l|l|l|}
\hline \multirow{2}{*}{ Repertorio Discursivo } & Archipiélago de significado & Formas gráficas \\
\hline \multirow{2}{*}{ Justificación } & Fuga & Fuga camino mas fácil \\
\cline { 2 - 3 } & Debilidad & Sin carácter, influenciable \\
\hline \multirow{2}{*}{ Juicio } & Normalidad & Normal \\
\cline { 2 - 3 } & Necesidad & $\begin{array}{l}\text { Tiene necesidad de, con } \\
\text { problemas }\end{array}$ \\
\hline Comparación & Caso específico & No se puede juzgar, depende \\
\hline
\end{tabular}

Tab. 5. Repertorios discursivos

A través del repertorio de la justificación, las mujeres entrevistadas activan un proceso dirigido a explicar las razones que están en la base del comportamiento de 
La construcción de la identidad de la mujer consumidora de sustancias ilegales...

consumo de sustancias. En concreto (en términos de contenido), utilizan el archipiélago de la fuga caracterizado por las formas gráficas "escapar" y "camino más fácil” y el archipiélago de la “debilidad” caracterizado de las formas gráficas "sin carácter” y “condicionable". Además, el uso de la modalidad cognoscitiva del juicio, evidencia cómo las entrevistadas activan teorías personales en la descripción de la mujer tóxicodependiente y que lo hacen recurriendo a contenidos relativos a una presunta normalidad, evidenciable por el uso del archipiélago de significado de la normalidad y del archipiélago de la necesidad, caracterizado por las formas gráficas "tiene necesidad de" y "con problemas".

Del mismo modo, emerge el uso del repertorio de la comparación, entre diferentes elementos, a través del archipiélago del caso específico caracterizado de las formas gráficas "no se puede juzgar" y "caso específico".

En referencia a las variables consideradas, es posible destacar cómo el grupo de personas tóxico-dependientes utilizan de modo característico el repertorio de la justificación y del juicio, este último usado de modo particular incluso por el grupo de las personas no consumidoras.

\subsection{Descripción de las respuestas hechas frente a la pregunta “¿Cómo describirías una embarazada tóxico-dependiente?”}

La pregunta pretende describir las modalidades cognoscitivas utilizadas por las mujeres entrevistadas respecto a la descripción de una mujer tóxico-dependiente embarazada.

A partir del análisis del texto de las respuestas hechas, se evidencia cómo las entrevistadas utilizan 2 repertorios discursivos, el repertorio del juicio y el repertorio de la justificación, y 4 archipiélagos de significado como se resume en la siguiente tabla. 


\begin{tabular}{|l|l|l|}
\hline \multirow{2}{*}{ Repertorio Discursivo } & Archipiélago de significado & Formas gráficas \\
\hline \multirow{2}{*}{ Juicio } & Necesidad de ayuda & De ayudar, de tutela \\
\cline { 2 - 3 } & Salud & Meter en peligro, vida, loca \\
\cline { 2 - 3 } & Irresponsabilidad & $\begin{array}{l}\text { Egoísta inmadura } \\
\text { Justifímulo, forma de rescate } \\
\text { para salir de la droga }\end{array}$ \\
\hline
\end{tabular}

Tab. 6. Repertorios discursivos

El uso de la modalidad cognoscitiva del juicio muestra cómo las entrevistadas activan teorías personales en la descripción de la mujer tóxico-dependiente durante el embarazo y cómo, en términos de contenido, lo hacen a través de los archipiélagos de la necesidad de ayuda, caracterizado por las formas gráficas “de ayudar", "de tutelar", a través del archipiélago de la salud, caracterizado por las formas gráficas "meter en peligro", "vida", "loca" y por repertorio de la inmadurez, caracterizado por las formas gráficas “egoísta” e "inmadura”. Además, a través del repertorio de la justificación, las mujeres entrevistadas activaron un proceso que tendía a individualizar y a dar las razones de base en el comportamiento del consumo de sustancias. En concreto (en términos de contenido), durante el embarazo las mujeres embarazadas ven una oportunidad de "rescate" (salida), como se deduce del uso de los archipiélagos de la oportunidad caracterizado de las formas gráficas "estímulo", "formas de rescate", "para salir de la droga".

En relación a las variables consideradas, se nota cómo el grupo de personas tóxico-dependientes utilizan de modo característico el repertorio del juicio, con particular referencia al archipiélago de la salud, mientras que el grupo de las personas no consumidoras utilizan de modo característico el repertorio de juicio en el archipiélago de la necesidad de ayuda y el repertorio de la justificación.

\subsection{Descripción de las respuestas realizadas a la pregunta “¿Cómo piensas que la gente común describiría a una mujer tóxico-dependiente?”}


La construcción de la identidad de la mujer consumidora de sustancias ilegales...

La pregunta propuesta pretendía conocer la teoría de las personas entrevistadas, en lo que respecta a la descripción de una mujer tóxico-dependiente, entre las posibles respuestas de sentido común.

En el análisis del texto de las respuestas a dicha pregunta se observa cómo las entrevistadas usan 2 repertorios discursivos: el repertorio del juicio y el repertorio de la comparación, y 3 archipiélagos de significado, como se observa en la siguiente tabla.

\begin{tabular}{|l|l|l|}
\hline \multirow{2}{*}{ Repertorio Discursivo } & Archipiélago de significado & Formas gráficas \\
\hline \multirow{2}{*}{ Juicio } & Necesidad de ayuda & $\begin{array}{l}\text { Tiene problemas, necesita } \\
\text { ayuda }\end{array}$ \\
\cline { 2 - 3 } & Fragilidad & Frágil, se ha equivocado \\
\hline Comparación & Consumo & Depende qué sustancia se usa \\
\hline
\end{tabular}

Tab. 7. Repertorios discursivos

El uso del repertorio del juicio pone de manifiesto cómo las entrevistadas atribuyen a las personas en general una modalidad discursiva centrada en el uso de teorías personales y juicios de valor, que en lo específico (en términos de contenido) hacen referencia a temas ligados al archipiélago de la necesidad de ayuda, caracterizado de las formas gráficas "frágil” y "se ha equivocado".

Paralelamente atribuyen a las personas en general descripciones construidas a partir del repertorio de la comparación, o sea, les atribuyen modalidades de definición que generan una contraposición entre diversos elementos, $y$ en concreto, referidos a temáticas asociadas al tipo de consumo.

En referencia a las variables consideradas, es posible destacar cómo el grupo de las personas tóxico-dependientes utilizan de manera característica el repertorio de la comparación, mientras el grupo de las personas no consumidoras utilizan de modo característico el repertorio del juicio.

\subsection{Descripción de las respuestas frente a la pregunta “¿Cómo piensa que la gente común describiría una mujer embarazada tóxico-dependiente?”}


La pregunta realizada trataba de mostrar la teoría de las personas entrevistadas respecto a la descripción de una mujer tóxico-dependiente embarazada entre las posibles respuestas de sentido común.

En el análisis del texto de las respuestas a la pregunta realizada se observa cómo las entrevistadas utilizan 2 repertorios discursivos, el del juicio y el de la justificación y 5 archipiélagos de significado, tal y como se recoge en la siguiente tabla.

\begin{tabular}{|l|l|l|}
\hline \multirow{2}{*}{ Repertorio Discursivo } & Archipiélago de significado & Formas gráficas \\
\hline \multirow{3}{*}{ Juicio } & Indiferencia & Indiferentes, la desdeñan \\
\cline { 2 - 3 } & Crítica & La critican, desaprobación \\
\cline { 2 - 3 } & Interrupción del embarazo & $\begin{array}{l}\text { Debe abortar, no tiene } \\
\text { derecho }\end{array}$ \\
\hline Justificación & Irresponsabilidad & Irracional, no se da cuenta \\
\hline & Reacción & Modo de reaccionar \\
\hline
\end{tabular}

Tab. 8. Repertorios discursivos

El uso del juicio evidencia cómo las entrevistadas atribuyen a las personas en general modalidades discursivas centradas en la utilización de teorías personales y juicios de valor que específicamente, en términos de contenido, hacen referencia a contenidos ligados al archipiélago de la indiferencia, caracterizado por las formas gráficas "indiferente" y "la desdeñan", al archipiélago de la crítica, a través de las formas gráficas "la critican” y "desaprobación”, al archipiélago de la interrupción del embarazo y al de la irresponsabilidad, caracterizados respectivamente de las formas gráficas "debe abortar", "no tiene derecho" y de "irracional" y "no se da cuenta".

Además, atribuyen descripciones construidas a partir del repertorio de la justificación, a temáticas relacionadas específicamente con el archipiélago de la reacción, caracterizado con la forma gráfica "modo de reaccionar".

En referencia a las variables consideradas, es posible evidenciar cómo el grupo de las mujeres embarazadas utilizan de manera característica el repertorio del juicio. 
La construcción de la identidad de la mujer consumidora de sustancias ilegales...

\subsection{Descripción de las respuestas realizadas frente a la pregunta “¿Cómo piensas que tus familiares te describirían como mujer?”}

La pregunta presentada pretende mostrar las teorías de las personas entrevistadas respecto a la descripción que harían de ellas los propios familiares.

A partir del análisis del texto de las respuestas realizadas respecto a la pregunta, se observa cómo las entrevistadas usan 1 repertorio discursivo, el del juicio, y 2 archipiélagos de significado, como se puede observar de forma resumida, en la siguiente figura.

\begin{tabular}{|l|l|l|}
\hline \multirow{2}{*}{ Repertorio Discursivo } & Archipiélago de significado & Formas gráficas \\
\hline Juicio & Fragilidad & Frágil, todavía una niña \\
\cline { 2 - 3 } & Inseguridad & Insegura, necesidad de \\
\hline
\end{tabular}

Tab. 9. Repertorio discursivo

A través del repertorio del juicio, las mujeres entrevistadas atribuyen a sus propios familiares definiciones de sí mismas generadas a partir de teorías personales, que en términos de contenido se refieren al archipiélago de la fragilidad, caracterizado por las formas gráficas "frágil”, "todavía una niña” y al archipiélago de la inseguridad, caracterizado por las formas gráficas "insegura" y "necesidad de cuidados".

En relación a las variables consideradas, se observa que los discursos producidos no caracterizan a ninguno de los grupos entrevistados, y sin embargo pertenecen indistintamente a todas las personas entrevistadas.

4.10. Descripción de las respuestas entregadas de acuerdo a la pregunta ¿“Cómo piensas que tus familiares te describirían como persona consumidora de sustancias?"

La pregunta propuesta al grupo de personas consumidoras de sustancias intenta mostrar las teorías de las personas entrevistadas respecto a cómo creen que las describen sus propios familiares. 
A partir del análisis del texto de las respuestas surge el uso de 2 repertorios discursivos por parte de las entrevistadas, el repertorio del juicio y el repertorio de la justificación y 4 archipiélagos de significado, tal y como muestra la siguiente tabla.

\begin{tabular}{|l|l|l|}
\hline \multirow{2}{*}{ Repertorio Discursivo } & Archipiélago de significado & Formas gráficas \\
\hline \multirow{2}{*}{ Juicio } & Desconfianza & No confían, dispuestas a todo \\
\cline { 2 - 3 } & Delincuencia & Mentirosa delincuente \\
\hline \multirow{2}{*}{ Justificación } & Necesidad & Necesitada, influenciable \\
\cline { 2 - 3 } & Problema & Problema doble \\
\hline
\end{tabular}

Tab.10. Repertorios discursivos

Utilizando el repertorio del juicio, las mujeres entrevistadas atribuyen a los propios familiares definiciones de sí mismas generadas a partir de teorías personales, que en términos de contenido se refieren al archipiélago de la desconfianza, caracterizado por las formas gráficas "no se fían”, "dispuestas a todo" y al archipiélago de la delincuencia, caracterizado de las formas gráficas: "mentirosa" y "delincuente". Además, con el repertorio de la justificación, atribuyen a la propia familia definiciones generadas a partir de la modalidad discursiva, orientadas a la búsqueda de la razón en cuanto al comportamiento de consumo de sustancias, en particular a través del uso de contenidos que se refieren a los archipiélagos de la necesidad, caracterizado de las formas gráficas "necesitada", "influenciable" y del archipiélago de los problemas, caracterizado de las formas gráficas "problema" y "débil".

En referencia a las variables consideradas, es posible observar cómo los discursos producidos no caracterizan a ningún grupo entrevistado y por tanto, no se evidencia una diferencia significativa entre las mujeres tóxico-dependientes y las mujeres tóxico-dependientes embarazadas.

\subsection{Descripción de las respuestas entregadas ante la pregunta “CCómo piensas que tus familiares te describirían embarazada?”}


La construcción de la identidad de la mujer consumidora de sustancias ilegales...

La pregunta presentada intenta revelar las teorías de las personas entrevistadas respecto a cómo creen que serían descritas por sus propios familiares, como mujer en estado de embarazo.

A partir del análisis del texto de las respuestas generadas en esta pregunta, las entrevistadas utilizan 1 repertorio discursivo, el del juicio, y 3 archipiélagos de significado que se observan en la siguiente tabla.

\begin{tabular}{|l|l|l|}
\hline \multirow{2}{*}{ Repertorio Discursivo } & Archipiélago de significado & Formas gráficas \\
\hline \multirow{2}{*}{ Juicio } & Fragilidad & $\begin{array}{l}\text { Fragilidad, flor que se está } \\
\text { abriendo }\end{array}$ \\
\cline { 2 - 3 } & Desinterés & No le interesa \\
\cline { 2 - 3 } & Normalidad & Como todos, normalmente \\
\hline \multicolumn{2}{|c|}{} & Tab.11. Repertorio discursivo
\end{tabular}

Mediante el repertorio del juicio, las mujeres entrevistadas atribuyen a los propios familiares definiciones de sí mismas generadas a partir de teorías personales, que en términos de contenido hacen referencia al archipiélago de la fragilidad, caracterizado por las formas gráficas "fragilidad" y al de "flor que se está abriendo", al archipiélago del desinterés caracterizado por formas gráficas como "no le interesa", así como del archipiélago de la normalidad, caracterizado de formas gráficas como "como todas" y "normalmente".

Respecto a las variables consideradas, es posible evidenciar cómo las mujeres consumidoras de sustancias se refieran de modo característico al tema de la normalidad.

\section{Consideraciones finales}

De los resultados descritos en el párrafo precedente, se pueden concluir una serie de consideraciones finales. 
Es posible mostrar que el evento del embarazo no constituye un elemento capaz de determinar un cambio en la modalidad cognoscitiva utilizada por las mujeres consumidoras de sustancias ilegales. En las respuestas a las preguntas que no hacen una referencia explícita al comportamiento de consumo, o sea, donde se solicitaba sólo una descripción de sí misma como persona, como madre en el futuro y como embarazada, se observa el uso común del repertorio del juicio para los grupos investigados; además, el repertorio de la proyección en el futuro se muestra característico del grupo de mujeres embarazadas, sin que se observe diferencia alguna en relación al comportamiento de consumo.

En cuanto a las respuestas a la pregunta que se refería concretamente al comportamiento de consumo de sustancias consideradas ilegales, se observa que al dar una definición de mujer tóxico-dependiente las entrevistadas utilizan indistintamente los repertorios de juicio y de la comparación; así mismo se revela como característico de las mujeres consumidoras el repertorio de la justificación. Del mismo modo, al responder a la pregunta que pedía una definición de la mujer consumidora en estado de embarazo, las mujeres consumidoras utilizan de manera característica el repertorio del juicio, mientras que el repertorio de la justificación es utilizado principalmente por las mujeres no consumidoras. No se puede destacar una diferencia entre la modalidad utilizada por las mujeres consumidoras en relación al estado de embarazo.

Respecto a las preguntas que pedían a las entrevistadas imaginar la descripción que la gente en general podría hacerse de las mujeres consumidoras de sustancias y consumidoras en estado de embarazo, se observa que las mujeres consumidoras utilizan más el repertorio del juicio que el repertorio de la comparación, usado por parte del grupo de las mujeres no consumidoras. En la definición de la mujer tóxico-dependiente embarazada y el uso común del repertorio de la justificación, se revela el uso característico sin distinción del repertorio del juicio por parte de las mujeres embarazadas respecto al consumo de sustancias. 
La construcción de la identidad de la mujer consumidora de sustancias ilegales...

Finalmente, en cuanto a las preguntas que pedían imaginar la descripción que la propia familia haría de la mujer, como mujer consumidora de sustancias y como mujer embarazada, se observa el uso común del repertorio del juicio.

Por tanto, es posible afirmar que un estado de embarazo constituye un momento crítico en la vida de las mujeres no consumidoras entrevistadas. Esto supone una novedad, respecto a lo comúnmente aportado por la literatura, donde el período de embarazo de las mujeres consumidoras de sustancias ilegales se considera como una ocasión para reorganizar la propia identidad de manera particular. Sin embargo, en la presente investigación se muestra que, si bien durante el embarazo son reconocibles ciertas diferencias, en los discursos producidos por las mujeres consumidoras de sustancias, éstas son atribuibles a aspectos de contenido pero no al proceso cognoscitivo activado y por tanto no atribuible a procesos de generación, construcción y mantenimiento de la propia identidad. 


\section{BIBLIOGRAFÍA}

Fava-Viziello, G. y Stocco P. (1997): Tra genitori e figli, la tossicodipendenza. Milano, Mason.

Malagoli-Togliatti M. y Mazzoni S. (1993): Maternità e tossicodipendenza. Milano, Giuffrè.

Turchi G. P. (coord.) (2002): Tossicodipendenza. Generare il cambiamento tra mutamento di paradigma ed effetti pragmatici. Upsel, Padova.

Turchi G. P. (2002): “Il Modello Narrativistico", en S. Marhaba (2002): Introduzione alla psicologia. Upsel, Padova.

Turchi G. P. y Della Torre C. (2006): Psicologia de la Salute (Pubblicazione a cura di Ministero della Giustizia, Istituto Superiore Studi Penitenziari.

-(2005): Progetto Chitone: Reato e identità, Atti e contributi per la formazione e l'operatività professionale. Upsel, Padova.

Agazzi E., Buzzoni M. y Servalli G. (corrds.) (1987): Linguaggio comune e linguaggio scientifico. Franco Angeli, Milano.

Agazzi E. (1972): I contenuti dell'apprendimento scolastico secondario e il problema dell'interdisciplinarietà, in AA.VV. (1972): La comunità scolastica. Roma. 